\title{
A Comparative Study of a Digital Modified Internal Model Control and RST Regulators Applied on a Permanent Magnet Synchronous Machine (PMSM) Powered by Voltage Inverter
}

\author{
Jamel Khedri and Mansour Souissi \\ Automatic Control Unit, National School of Engineers of Sfax, ENIS \\ Road of soukra, km 3.5 - BP.W, 3038, Sfax, Tunisia \\ khedrijamel@yahoo.fr
}

\begin{abstract}
For control systems, nonlinearity, parameters variations and noise always represent a major disadvantage. Most classical control such that vector control, which stay the most frequently used and the most widespread, fail to reach high control performance under these conditions. In the present paper, an application of speed regulation to a permanent magnet synchronous machine (PMSM) where a modified internal model control and RST controllers are proposed. Both regulators are carried out under parameters variations and load disturbance. A comparative evaluation is conducted between the two controllers. Experimental results are satisfactory.
\end{abstract}

Keywords: Modified internal reference model, RST, parameters variations, PMSM.

\section{Introduction}

Permanent magnet synchronous machine remains the most frequently used in industrial application among several variety of ac motors. This is due to many considerations such as its simplicity of design, high air-gap flux density, high energy efficiency, low maintenance, cost and size. Nonetheless, the PMSM is nonlinear with multiple coupled states and it is affected by parameters variations and external disturbance [1]. This makes difficult to obtain a satisfactory high performance with linear control approach. For this purpose, with the growth of modern control algorithm and machine control, several control methods have been implemented especially robust control [2], [3] optimal control [4], adaptive control [5],[6], [7], nonlinear control [8], sliding model control [9] and intelligent control [10].These control methods allow high performance and enrich PMSM control strategy. Despite, such control methods lead to good performance criteria, industrial applications are implemented via easier algorithms because they are simple to be developed in microcontrollers and provide practical solutions.

This paper suggests the comparison of performances given with the two very habitual algorithms based on a Modified Internal Model Control and an RST controller.

IMC has been introduced for the first time in 1982 by Morari and Garcia for process control applications. It have the capability of disturbance rejection, tracking and provide efficient high performance in terms of stability and robustness [11], [12]. During the past decades especially in 1989 this model control is extended to motor control systems [13] by the regulation of the current of ac motors. In 2005 shao and al was applied an adaptive IMC algorithm to control the speed of a PMSM. As well as most of control law, IMC controller has disadvantages especially it cannot give a good load disturbance rejection mainly when the desired closed-loop dynamics are much faster than the process one [14]. Besides IMC control method does not take into account the control input saturation which can degrade the control performance. Thus a modified internal model control method will be necessary [15].

RST controller which has been appeared in 1980 is an extension to classical series compensator. It is a two degree of freedom (2DOF) regulator, whose synthesis is notably algebraic based on pole placement method. The polynomial $\mathrm{R}$ and $\mathrm{S}$ leads to create a feedback control in order to become robust to uncertainties, whereas the polynomial $\mathrm{T}$ is injected in the feedforward to prove the tracking trajectory of reference [16]. Recently, RST controller is devised to induction

Received: January $18^{\text {th }}, 2017$. Accepted: March $30^{\text {th }}, 2018$

DOI: $10.15676 /$ ijeei.2018.10.1.8 
motor [17] and PMSM [18] where the knowledge of the dynamic of the model of the physical system is necessary. This method of control is characterized by pliability and simplicity in designing. Besides it demonstrate also good results in terms of tracking and regulation while ensuring stability and robustness of the system.

This work propounds a comparison of the performances given with the two very widespread algorithms namely, the Internal Model Controller and the RST controller.

For a judicious comparison, and in order to obtain a high load disturbance rejection the RST controller will be coupled to a load torque observer in order to re-estimate the speed and reconstruct the load torque. The presence of the observer ensures a generation of an adequate current allowing to anticipate the disturbances and to reduce the undulations of the torque.

The paper is organized as follows: in section 2, a nonlinear state model of the PMSM is briefly presented. Besides a block scheme of the control structure is given while presenting the regulation of the direct and the quadrate stator currents by a classical PI regulator. In section 3 and 4, the design of a modified IMC and digital RST controllers for the PMSM speed regulation are described in detail. The last section is dedicated to experimental results applied to the PMSM while comparing the performances given by each of the two developed methods.

\section{Nonlinear state model of the PMSM}

By referring to classical simplifying assumptions, the stator $d-q$ set of equations of the PMSM in the rotating rotor reference frame [19] are given as follows:

$$
\begin{aligned}
& \frac{d I_{d}}{d t}=-\frac{R_{s}}{L_{d}} I_{d}-\frac{L_{q}}{L_{d}} w_{r} I_{q}+\frac{1}{L_{d}} v_{d} \\
& \frac{d I_{q}}{d t}=-\frac{R_{s}}{L_{q}} I_{q}-\frac{L_{d}}{L_{q}} w_{r} I_{q}-\frac{\phi}{L_{q}} w_{r}+\frac{1}{L_{q}} v_{q} \\
& \frac{d w_{r}}{d t}=\frac{p\left(c_{e}-c_{r}\right)}{J}-\frac{f_{c}}{J} w_{r} \\
& c_{e}=p \phi I_{q} \\
& \frac{d w_{r}}{d t}=\frac{p^{2} \phi}{J} I_{q}-\frac{f_{c}}{J} w_{r}-\frac{p}{J} c_{r}
\end{aligned}
$$

where $v d, v q$ are d-q axis stator voltages, $I_{d}, I_{q}$ are d-q axis stator currents, $L_{d}, L_{q}$ are the d-q axis inductances, $R_{s}$ is the stator winding resistance, $\phi$ is the flux linkage of the permanent magnets, $w_{r}$ is the angular velocity of the motor shaft, $f_{c}$ is the friction coefficient relating to the rotor speed; $J$ is the moment of inertia of the rotor, $c_{e}$ is the electromagnetic torque, $c_{r}$ is the external load torque and $p$ is the number of pole .

By analyzing the set of equation (1) we can note that the model of the PMSM is strongly coupled and leads us to raise that it is nonlinear. The most adopted strategy to compensate the nonlinearities and getting a model similar to a DC motor consists in keeping the direct current $I_{d}$ at the value zero. Besides for a smooth pole machine, the good choice of its operation is obtained by limiting the stator currents to the only component $I_{q}$ [20]. The design of the applied control structure to the studied PMSM is given by the block scheme of figure 1 . 


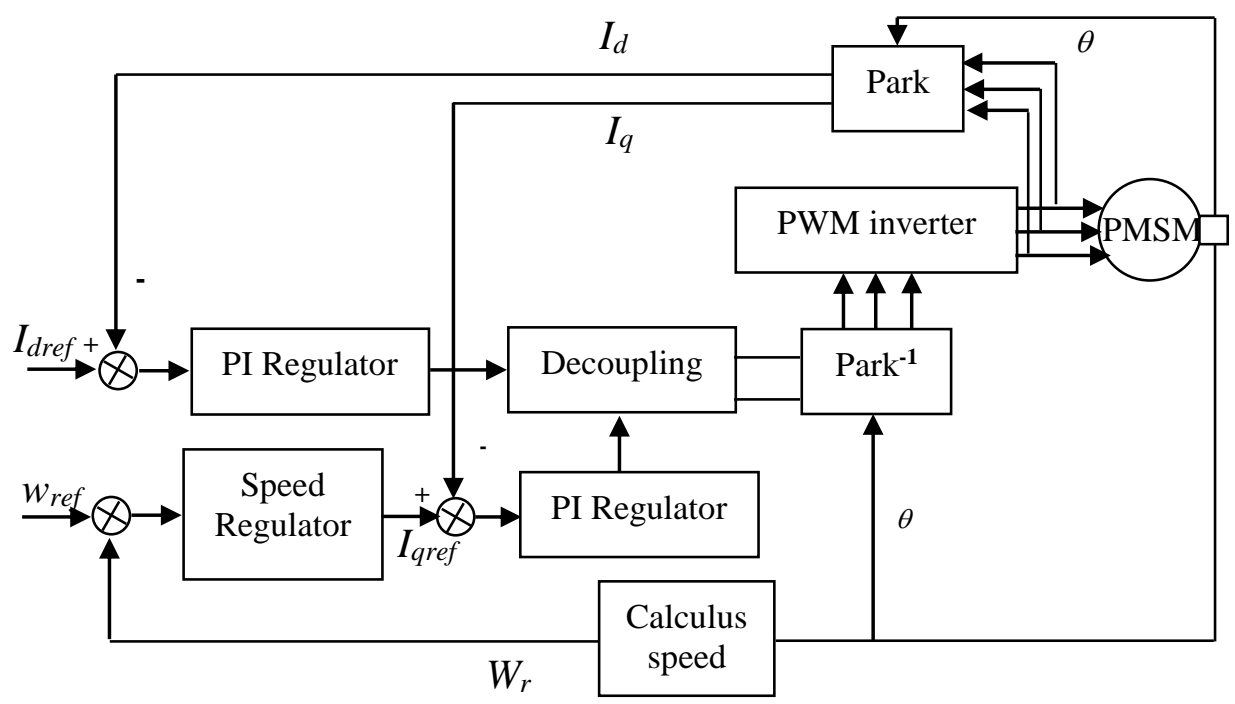

Figure 1. Block scheme of control structure

In this control structure, the stator currents $I_{d, q}$ are controlled by classical PI regulators since their dynamics are a first order. Moreover, since we have a smooth machine, we can impose the same parameters $K_{p}$ and $K_{i}$ of the regulators for the currents $I_{d}$ and $I_{q}$. The block diagram current is as follow:

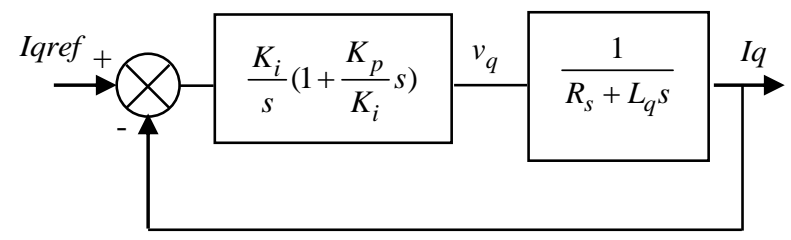

Figure 2. Current control by PI

These parameters will be set by the compensation of the zero introduced by each of the two regulators by the dynamics of the currents [21]. Therefore, the parameters of tuning are given by:

$$
\begin{gathered}
K_{p}=K_{i} \tau_{0} \\
K_{i}=\beta \frac{R_{s}}{\tau_{0}}
\end{gathered}
$$

Where $\tau_{0}=\frac{L_{q}}{R_{s}}$ is the time constant of the electric part, $\beta$ defines of the acceleration of the closed-loop current dynamic and characterize the desired dynamic in relation to real dynamic. In our study we chose a desired dynamic ten times faster then the real dynamic. Thus $\beta=0.1$

\section{IMC structure}

Standard internal model control structure illustrated by figure 3 consists of the given process to be controlled $H(s)$, the model process $\tilde{H}(s)$ and the internal model controller 
$C(s)$. The difference between the process and its model outputs is used for feedback to reflect the impact of process mismodelling and disturbance

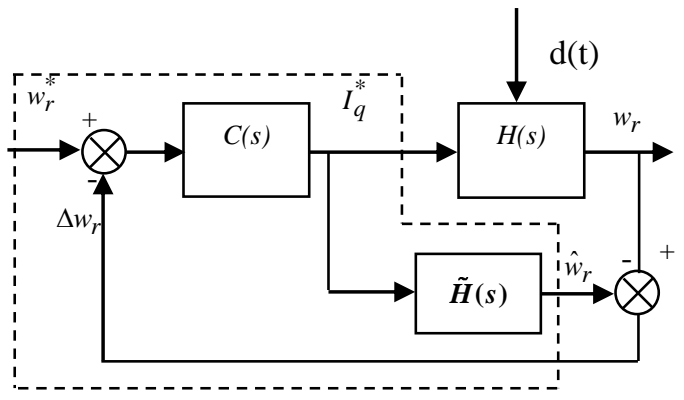

Figure 3. Standard IMC structure

From (1), we get:

$$
\begin{aligned}
\frac{d w_{r}}{d t} & =\frac{p^{2} \phi}{J} I_{q}-\frac{f_{c}}{J} w_{r}-\frac{p}{J} c_{r} \\
& =\frac{p^{2} \phi}{J} I_{q}^{*}-\frac{f_{c}}{J} w_{r}-\frac{p}{J} d(t)
\end{aligned}
$$

Where $d(t)=\frac{c_{r}}{p \phi}+\left(I_{q}^{*}-I_{q}\right)$, which contains the tracking error of the quadrate current loop $I_{q}$ and the load torque disturbance, $I_{q}^{*}, w_{r}{ }_{r}$ is the quadrate current and speed reference respectively.

As mentioned in section $2, I_{d}=0$ and for a zero load torque, equation (3) become:

$$
\frac{d w_{r}}{d t}=\frac{p^{2} \phi}{J} I_{q}-\frac{f_{c}}{J} w_{r}
$$

We can derive that the controller model of the PMSM is of the form [22]:

$$
H(s)=\frac{1}{a s+b}
$$

Where $a=\frac{J}{p \phi}, b=\frac{f_{c}}{p \phi}$

As we have a first order controlled model, the internal model is chosen as follows:

$$
\tilde{H}(s)=\frac{1}{\tilde{a} s+\tilde{b}}
$$

Where $\tilde{a}$ and $\tilde{b}$ are the internal model parameters.

The IMC control strategy requires the following assumptions:

1) We assume that the internal model is accurate, that is to say $H(s)=\tilde{H}(s)$. The closed loop stability of the system is ensured only if $H(s)$ and $C(s)$ each of them is stable [23].

2) In order to obtain a well tracking of the system to its input, we consider that $C(s)=H^{-1}(s)$ 
However, this ideal equality can not be obtained due to several reasons such that $H(s)$ cannot sometimes be invertible. Thus the internal model controller will be designed as follows:

$$
C(s)=\tilde{H}^{-1}(s) L(s)
$$

Where $L(s)$ is a low pass filter of the form:

$L(s)=\frac{1}{\varepsilon s+1}, \varepsilon$ denotes the time constant of filter.

From figure 2, we get:

$$
w_{r}(s)=\frac{C(s) H(s)}{1+C(s)[H(s)-\tilde{H}(s)]} w_{r}^{*}(s)-\frac{H(s)[1-C(s) \tilde{H}(s)]}{1+C(s)[H(s)-\tilde{H}(s)]} D(s)
$$

If $H(s)=\tilde{H}(s)$, then from (7) and (8), we get:

$$
w_{r}(s)=\frac{1}{\varepsilon s+1} w_{r}^{*}(s)-\frac{\varepsilon s}{(a s+b)(\varepsilon s+1)} D(s)
$$

From this last equation (9), it can be noticed that there are more interesting properties with regard to load disturbance rejection performance. This is due to the fact that the transfer function between $w_{r}(s)$ and $D(s)$ consists of $H(s)$. So the parameter $\varepsilon$ of the IMC filter $L(s)$ must be tuned perfectly. It is usually selected small enough to ensure load disturbance rejection performance. Another interesting property which must be taken into account is that the internal model controller output can exceed the saturation of $I_{q}^{*}$ in practical application. This is can affect the performance of system to be controlled [23]. Thus a modified IMC method will be necessary in order to get over the disadvantage of the standard IMC design and to ensure the capabilities of tracking and load disturbance rejection. To achieve this, a two-port IMC design is adopted [24] where a feedback controller $C_{1}(s)$ is added to the standard model control scheme as illustrated in figure 4.

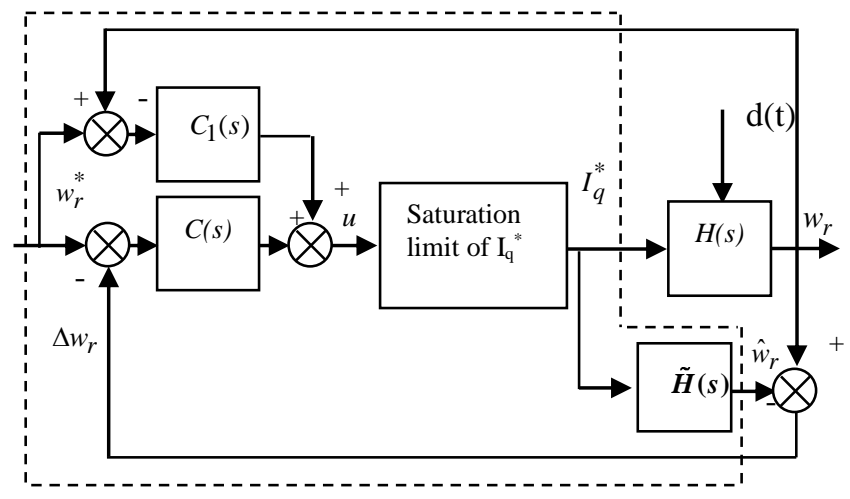

Modified IMC

Figure 4. Modified IMC structure of the PMSM

In this new structure, once output of modified IMC becomes saturated, the output of the controller $C_{1}(s)$ reacts as an antiwindup compensation and can compensate the effect of 
control input saturation and thus ensuring tracking performance. The saturation block presented in figure 4 is built based on amplitude limitation of the control input $u$. Thus we can derive:

$$
I_{q}^{*}=\left\{\begin{array}{l}
u,|u| \leq I_{q \max } \\
I_{q \max } \operatorname{sign}(u),|u| \geq I_{q \max }
\end{array}\right.
$$

From figure 4, we can derive:

$$
w_{r}(s)=\frac{\left[C(s)+C_{1}(s)\right] H(s)}{1+C(s)[H(s)-\tilde{H}(s)]+C_{1}(s) H(s)} w_{r}^{*}(s)-\frac{H(s)[1-C(s) \tilde{H}(s)]}{1+C(s)[H(s)-\tilde{H}(s)]+C_{1}(s) H(s)} D(s)
$$

To facilitate the study, the feedback controller $C_{1}(s)$ is considered as a constant term:

$$
C_{1}(s)=k
$$

In case of perfect internal model, i.e $H(s)=\tilde{H}(s)$, and According to (5), (7), (10) and (11), we get the following equation:

$$
w_{r}(s)=\frac{(k \varepsilon+a) s+k+b}{(a s+k+b)(\varepsilon s+1)} w_{r}^{*}(s)-\frac{\varepsilon s}{(a s+k+b)(\varepsilon s+1)} D(s)
$$

To ensure a good closed loop system tracking and load disturbance rejection performance, the term $k$ must settled perfectly in such a way to reduce the time constant. Thus compared to (9), we can derive:

$$
\frac{a}{k+b}<\frac{a}{b}
$$

Here, we present simulation results of the closed loop speed responses where controller's parameters are taken equal.

\section{$\mathrm{a}=0.0162$, b $0.0305, \mathrm{k}=0.19$ and $\varepsilon=0.01$}

Speed

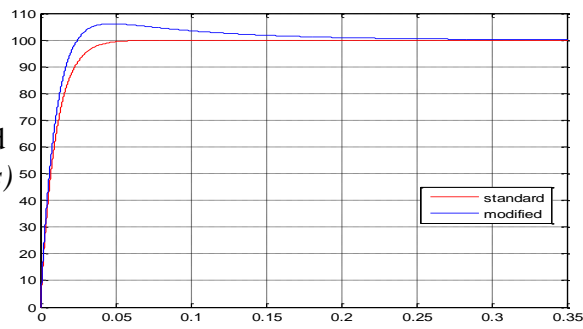

a) time $(\mathrm{s})$

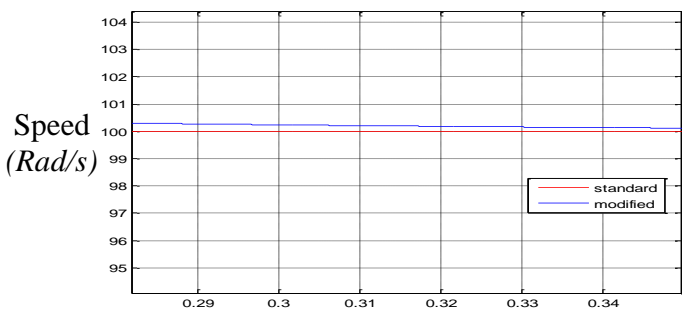

b)

time $(s)$

Figure 5. Speed responses of standard and modified IMC controllers (simulation).

a) speed, b) local curve of a)

Figure 5 a) show the response speed of the two controllers (standard and modified IMC). Results with modified IMC show a short overshoot and short settling time of $0.02 \mathrm{~s}$ while the standard one present a zero overshoot and a short settling time $(0.03 \mathrm{~s})$.

Figure $5 \mathrm{~b}$ ) is a partial enlargement of figure 5 a) on which we can notice that modified IMC method reduces steady state error more than standard IMC method.

Figure 6 present results of the two methods under load torque disturbance. To further obtain a fast speed response, we reduce the value of $\varepsilon$ as shown in figure 7 where $\varepsilon=0.005$. Settling time of the standard IMC method is almost equal to $0.0148 \mathrm{~s}$ and $0.01 \mathrm{~s}$ for the modified IMC. 


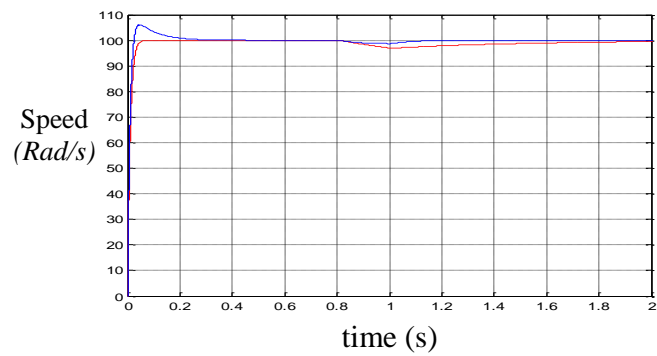

Figure 6. Speed responses of standard and modified IMC controllers under load torque disturbance (simulation)

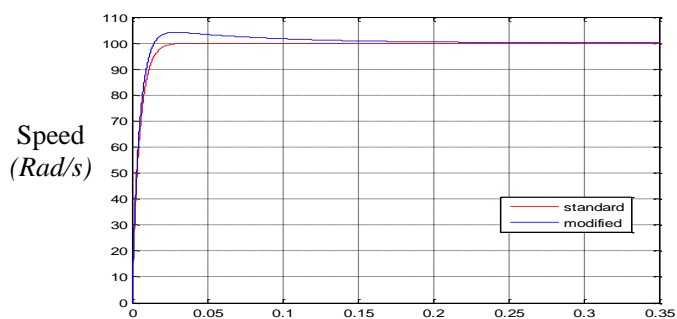

a) time $(s)$

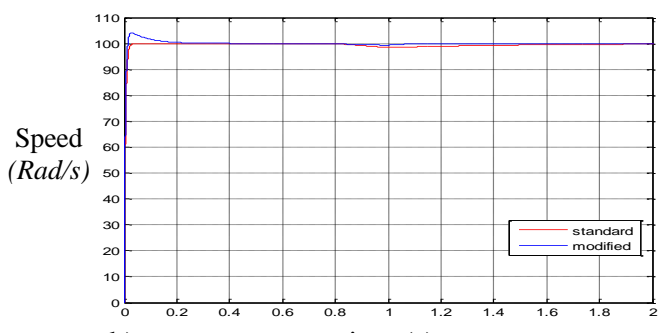

b) time $(s)$

Figure 7. Speed responses of standard and modified IMC controllers. a) speed, b) curve a) under load torque disturbance (simulation)

To more prove the above findings in terms of overshoot, settling time and steady state error, we present the step responses of the speed under the two methods for two different value of $\varepsilon$
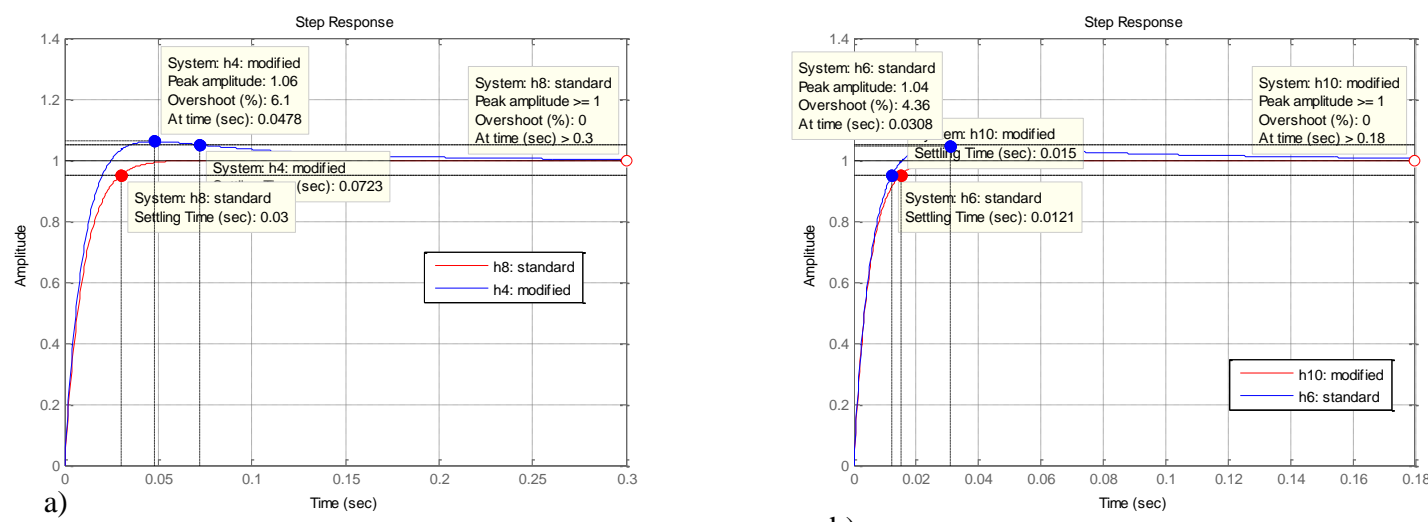

b)

Figure 8. speed step response time under standard and modified IMC methods. a) for $\varepsilon=0.01$, b) $\varepsilon=0.005$

\section{RST structure}

Digital RST controller structure shown in figure 8 is a two degrees of freedom which consists of three polynomials $R(s), S(s)$ and $T(s)$ to be finding. Its basic advantage is that it lets the user to choose independently the desired objectives [25] in terms of trajectory tracking by the polynomial $T(s)$ and regulation by $R(s)$ and $S(s)$. 


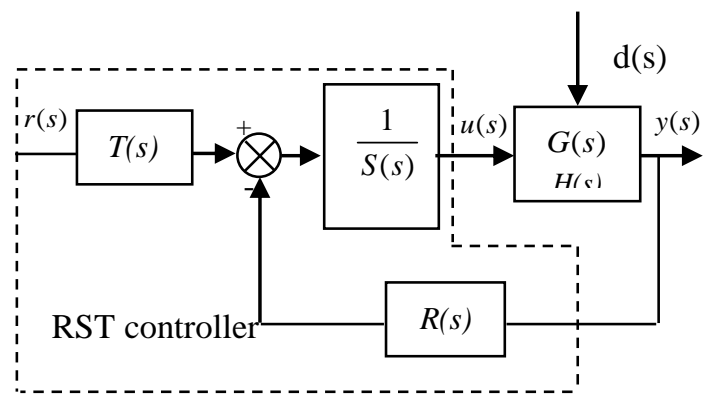

Figure 9. RST structure

From this structure, we can derive the control law $u(s)$ :

$$
u(s)=\frac{T(s)}{S(s)} r(s)-\frac{R(s)}{S(s)} y(s)
$$

Where $r(s), y(s)$ are the input and the output signal respectively.

The first step of the design consists in finding the polynomials $R(s)$ and $S(s)$ in such a way that the closed-loop transfer function from reference signal to output measured signal $\frac{y(s)}{r(s)}$ is equal to an imposed transfer function characterizing desired performances:

$$
G_{b f}(s)=\frac{B_{b f}(s)}{A_{b f}(s)}
$$

Let define the transfer function of the process $G(s)$ :

$$
G(s)=\frac{B(s)}{A(s)}
$$

From the block scheme in figure 9 , the closed-loop transfer function is given by:

$$
G_{b f}(s)=\frac{y(s)}{r(s)}=\frac{B(s) T(s)}{A(s) S(s)+B(s) R(s)}
$$

The identification between the closed-loop transfer function (16) and the desired one (14) give the following equation:

$$
\begin{aligned}
& A(s) S(s)+B(s) R(s)=A_{b f}(s) \\
& B(s) T(s)=B_{b f}(s)
\end{aligned}
$$

(17) can be transformed as follows:

$$
\begin{aligned}
& A(s) S(s)+B(s) R(s)=B(s) A_{b f}(s) \\
& T(s)=B_{b f}(s)
\end{aligned}
$$

To obtain $R(s)$ and $S(s)$, leads us to resolve the Diophantine equation (18) [26]:

$$
A(s) S(s)+B(s) R(s)=B(s) A_{b f}(s)
$$




\section{Remark 1:}

For a perfect regulation:

$$
\begin{aligned}
& \operatorname{deg}\left(A_{b f}(s)\right) \leq 2 \operatorname{deg}(A(s))-1 \\
& \operatorname{deg}(T(s)) \leq \operatorname{deg}\left(A_{b f}(s)\right)-\operatorname{deg}(A(s)) \\
& \operatorname{deg}(R(s)) \leq \operatorname{deg}\left(A_{b f}(s)\right)-\operatorname{deg}(A(s)) \\
& \operatorname{deg}(S(s)) \leq \operatorname{deg}(R(s))
\end{aligned}
$$

\section{A. Currents control}

As mentioned in section 2, the d-q axis currents loop structure have the same first order dynamics such as:

$$
G_{I d, q}(s)=\frac{B(s)}{A(s)}=\frac{1}{R_{s}+s L_{q}}=\frac{\frac{1}{R_{s}}}{1+s \tau_{e}}=\frac{\frac{1}{L_{q}}}{\frac{R_{s}}{L_{q}}+s}
$$

With $\tau_{e}=\frac{L_{q}}{R_{s}}$ is the electric time constant, $B(s)=\frac{1}{L_{q}}, A(s)=\frac{1}{s+\frac{R_{s}}{L_{q}}}$

Let us set $A_{b f}(s)$ and $B_{b f}(s)$ of the form:

$$
A_{b f}(s)=s+\frac{1}{\tau_{d}}, B_{b f}(s)=\frac{1}{\tau_{d}}
$$

$\tau_{d}$ refers to the electric time of the desired transfer function $G_{b f}(s)$.

The acceleration of the desired closed-loop currents dynamics is characterized by the relationship between

$\tau_{e}$ and $\tau_{d}$ such that:

$$
\tau_{d}=\frac{1}{\gamma} \tau_{e}
$$

In our study, $\gamma=10$ in order to obtain a desired dynamic ten times faster than the real one.

By referring to remark 1 , we can derive the different degrees of polynomials $R(s), S(s)$ and $T(s)$ such as:

$R(s)=r_{0}, S(s)=s_{0}$ and $T(s)=t_{0}$.

By solving (18) we get:

$$
R(s)=\frac{1}{\tau_{d}}-\frac{R_{s}}{L_{q}}, S(s)=\frac{1}{L_{q}} \text { and } T(s)=\frac{1}{\tau_{d}}
$$

\section{B. Speed control}

The design of the control structure is shown by the block scheme of figure 10 . 


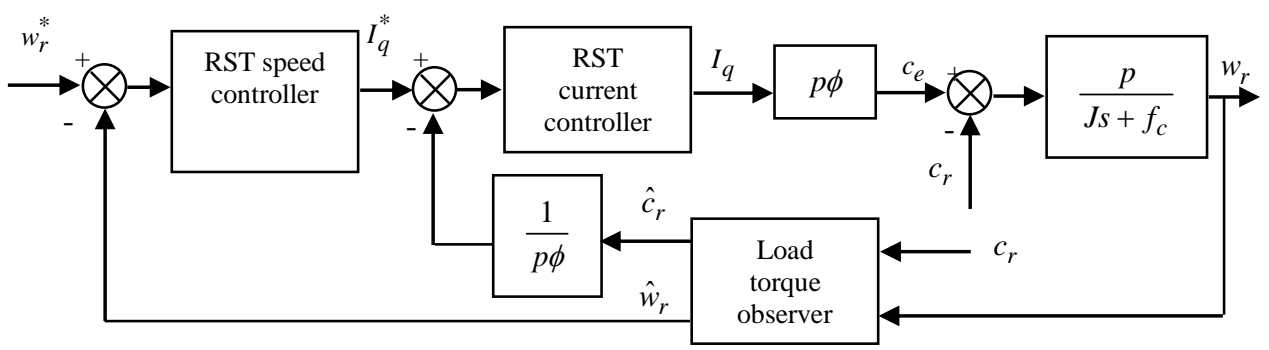

Figure 10. Block scheme of the proposed controller based load torque observer

This control structure consists of two blocks: an RST speed controller and a block of observer to estimate the load torque in order to reconstruct it by the reestimation of the speed. This is done in order to compensate substantially the disturbances due to load and parameters variations. Besides, the speed regulation output give a distorted image of the quadrate current $I_{q}$ which affects the system by oscillations. Thus the presence of the observer ensures a generation of an adequate current allowing to anticipate the disturbances and to reduce the undulations of the torque.

Before the design of the load torque observer, let itemize the speed correction.

From (1), and for a zero load torque, we can derive the transfer function of the speed loop:

$$
G_{w_{r}}(s)=\frac{p^{2} \phi_{f}}{J s+f_{c}}
$$

We assume a second order desired closed-loop transfer function of the form:

$$
G_{b f w_{r}}(s)=\frac{w_{n}^{2}}{s^{2}+2 \xi w_{n} s+w_{n}^{2}}
$$

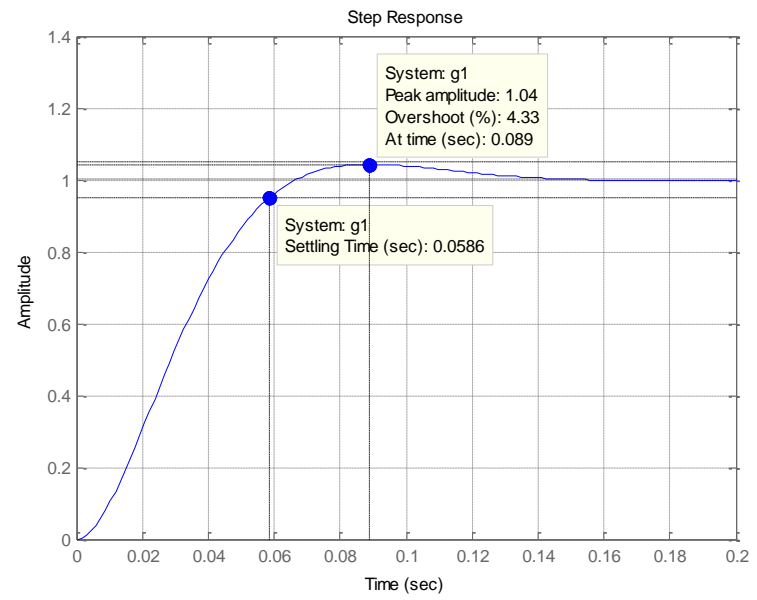

Figure 11. Speed step response time under RST methods

Remark1 leads to the polynomials $R(s), S(s)$ and $T(s)$ of the form:

$$
R(s)=r_{0}+r_{1} s, S(s)=s_{0}+s_{1} s \text { and } T(s)=t_{0}
$$

By using identity (18) we can derive the different coefficient: 


$$
r_{0}=t_{0}=w_{n}^{2}, s_{0}=1, s_{1}=\frac{p^{2} \phi_{f}}{J} \text { and } r_{1}=\frac{2 \xi w_{n} p^{2} \phi_{f}-J}{p^{2} \phi_{f}}
$$

$w_{n}=50, \xi=0.707$ in order to obtain a closed loop system response without overshoot and a lillte settling time.

\section{Load torque observer}

The observer is developed in order to estimate the speed and the load torque. Its structure is shown by the figure 10 on which the inputs are the load torque to be applied and the calculated speed derived from the numerical derivation position. The purpose of the observer is to provide an estimation error that converges quickly to zero than the system response.

Basing on matrix form and a load torque which varies slowly such that it is considered constant between two sampling steps, the observer equation is given by:

$$
\hat{\dot{x}}=A \hat{x}+B u+L C(x-\hat{x})
$$

Where $\hat{x}=\left(\begin{array}{l}\hat{w}_{r} \\ \hat{c}_{r}\end{array}\right)$ is the observed state, $L=\left[\begin{array}{ll}l_{1} & l_{2}\end{array}\right]^{T}$ is the observer gain matrix.

$$
A=\left[\begin{array}{cc}
-\frac{f_{c}}{J} & -\frac{p}{J} \\
0 & 0
\end{array}\right], B=\left[\begin{array}{c}
\frac{p^{2} \phi_{f}}{J} \\
0
\end{array}\right] \text { and } C=\left[\begin{array}{l}
1 \\
0
\end{array}\right]^{T}
$$

Let recall the estimation error $e=x-\hat{x}$

As mentioned above the goal of the observer is to ensure an estimation error that converges to zero the faster possible than the system response. Thus the error dynamic is given as follow:

$$
\dot{e}=\dot{x}-\hat{\dot{x}}=(A-L C) e
$$

Where $A-L C=\left[\begin{array}{cc}-\frac{f_{c}}{J}-l_{1} & -\frac{p}{J} \\ -l_{2} & 0\end{array}\right]$ is the observer matrix.

The stability of the error dynamic is governed by the observer matrix which must be Hurwitz. So the coefficients of the corresponding observer characteristic polynomial are then located at the adequate observer poles in order to ensure steady error estimation as quickly as possible. The characteristic polynomial is derived from $\operatorname{det}(s I-(A-L C))=s^{2}+\left(\frac{f_{c}}{J}+l_{1}\right) s+\frac{p}{J} l_{2}$

An adequate pole placement require the following condition:

$$
l_{1}>-\frac{f_{c}}{J}, l_{2}<0
$$

\section{Experimental results and interpretations}

In this part, to prove the effectiveness of the proposed methods, some experimental results were accomplished on a test benchmark which consists of two identical permanent magnet synchronous machines, one was considered as a motor and the other as generator (load), and they are equipped by incremental encoder and powered by a $127 \mathrm{v}$ continuous voltage inverter. The developed control algorithms were carried out via the software Matlab/SIMULINK and treated on Dspace 1104 card. The switching frequency is chosen equal to $10 \mathrm{kHz}$. The parameters of the two permanents magnet synchronous machines are shown in Table1 
Jamel Khedri, et al.

Table 1. parameters of the motor

\begin{tabular}{lc} 
Motor rated power $(\mathrm{kW})$ & 1 \\
Rated current $(\mathrm{A})$ & 6 \\
Pole pair number $(\mathrm{p})$ & 2 \\
Direct inductance $L_{d}(\mathrm{mH})$ & 4.5 \\
Quadrate inductance $L_{q}(\mathrm{mH})$ & 4.5 \\
Stator resistance $R_{s}(\Omega)$ & 0.57 \\
Rotor flux linkage $\phi(\mathrm{wb})$ & 0.64 \\
Motor inertia $J\left(\mathrm{Kg} \cdot \mathrm{m}^{2}\right)$ & 0.00208 \\
Viscous coefficient $f_{c}(\mathrm{~N} . \mathrm{m} . \mathrm{s} / \mathrm{rd})$ & 0.0039 \\
\hline
\end{tabular}

The test benchmark is given by figure 12 below

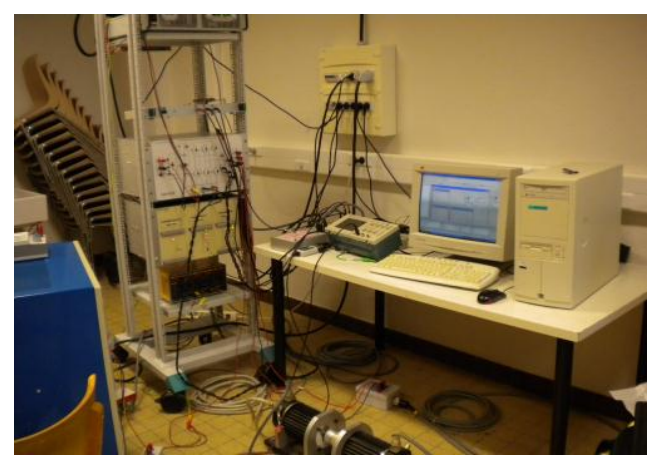

Figure 12. Photo of the test benchmark

Figure 13 represents respectively, the direct current $I_{d}$, the quadrate $I_{q}$, the speed tracking

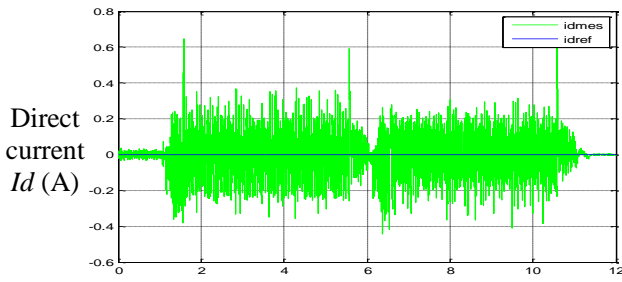

time $(s)$

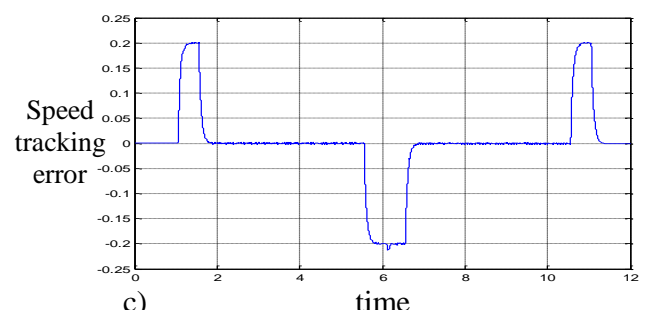

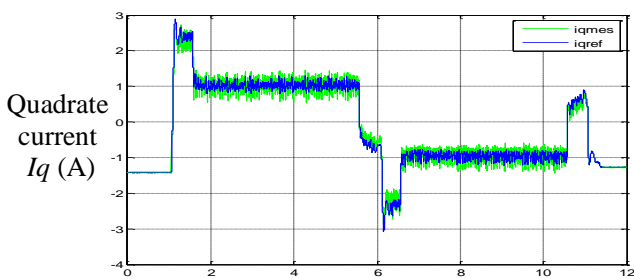

h) time $(\mathrm{c})$

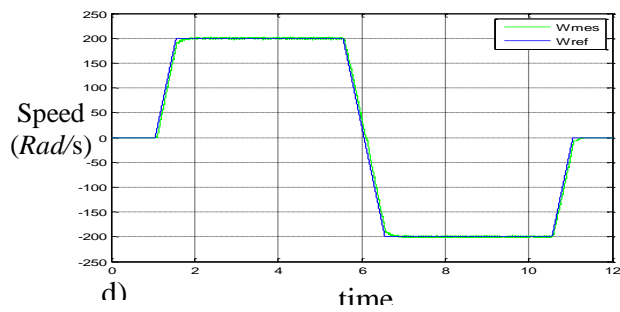

Figure 13. Experimental results under modified IMC method: a) $I_{d}$ measured current, b) $I_{q}$ measured, c) tracking speed error, d) measured speeds 
error and the speed versus time according to a profile of this last with a benchmark $(200,0$, $200 \mathrm{rd} / \mathrm{s}$ ) without load and under the modified IMC method. We notice that with this control method a good tracking speed and currents were obtained. Indeed, the speed converges to its reference with a 0.2 steady error and a practically without undershoot/overshoot. The same performances are achieved with a constant reference speed of $200 \mathrm{rd} / \mathrm{s}$ and under a load introduced at $t=2 s$ and cancelled à $t=9.8 s$ as shown in figure 14 .

Figures (15) and (16) show evolutions of the states above under the RST controller without and with load respectively. We observe that under this proposed method a quick convergences of measured states around the settled trajectories with rejection of disturbance.

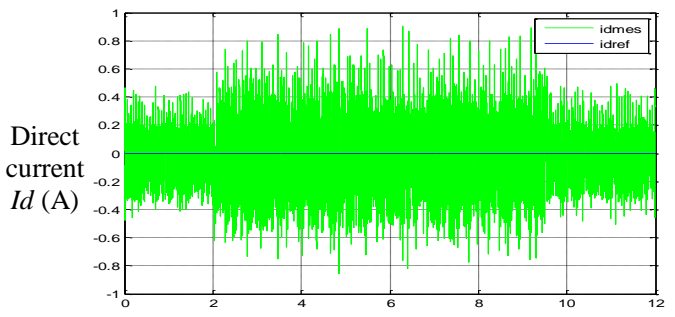

a)

time

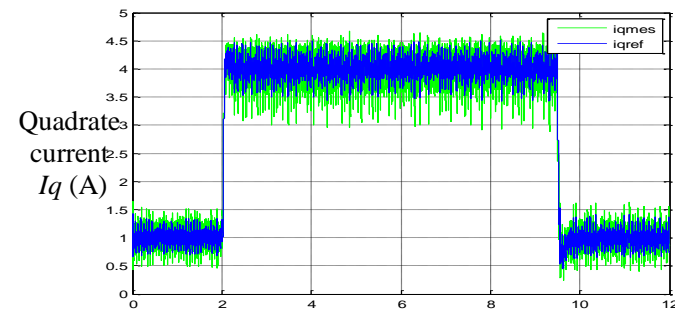

b) time (s)

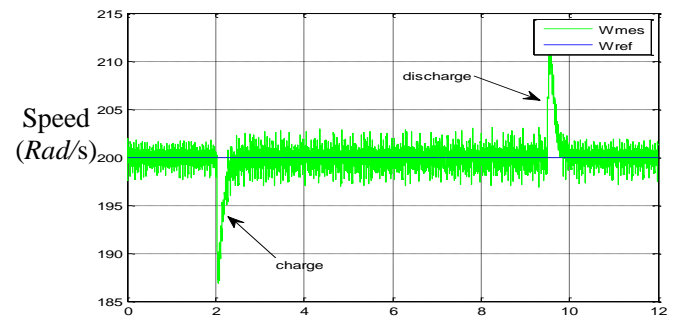

c) time (s)

Figure 14. Experimental results with modified IMC method: a) $I_{d}$ measured current, b) $I_{q}$ measured, c) measured speeds under load torque

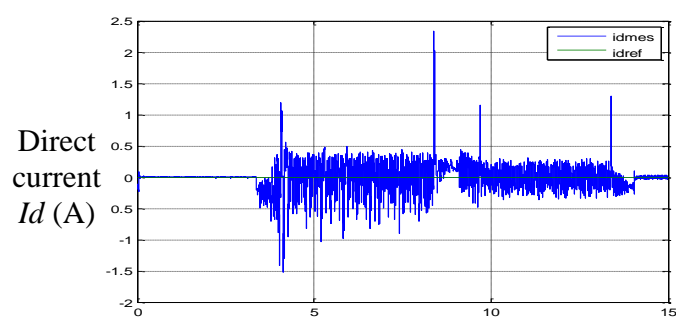

a)

time (s)

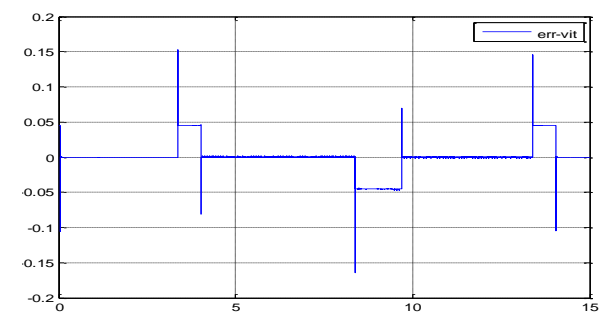

c) time (s)

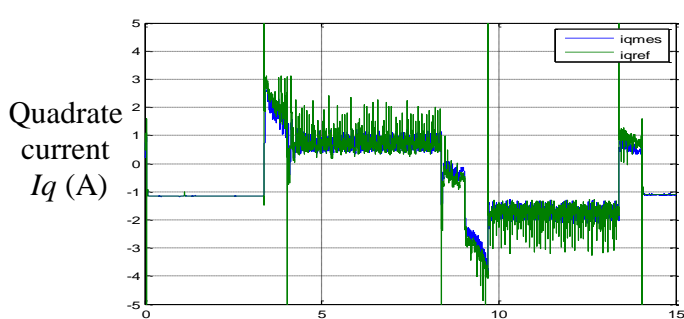

b)

time $(s)$

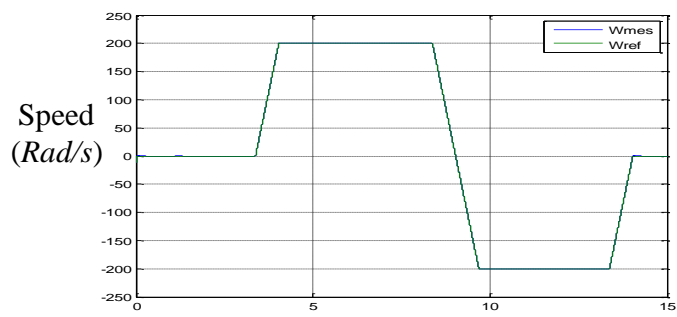

d)

time (s)

Figure 15. Experimental results under RST method: a) $I_{d}$ measured current, b) $I_{q}$ measured,

c) tracking speed error, e) measured speeds 


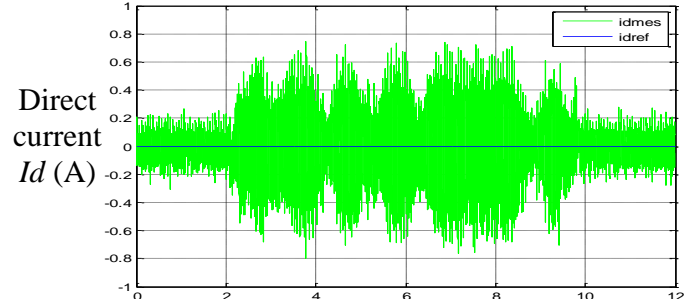

a)

time $(s)$

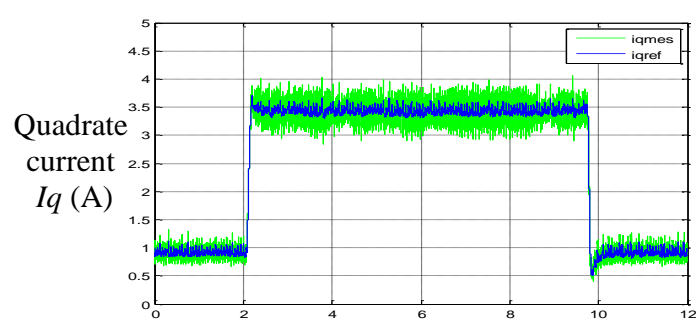

b)

time $(s)$

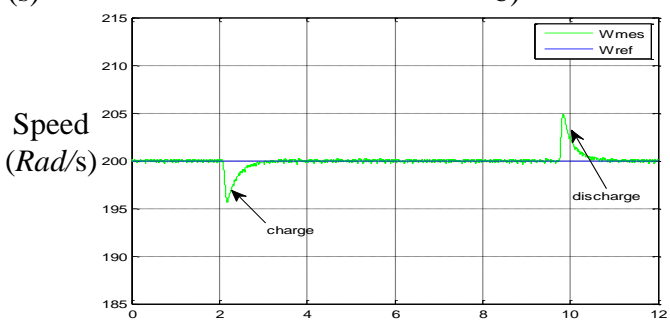

c) time (s)

Figure 16. Experimental results with RST method: a) $I_{d}$ measured current,

b) $I_{q}$ measured, c)measured speeds under load torque

\section{Robustness}

To test the robustness of the developed control methods especially in case of a bad assessment of electrical and mechanical parameters which can affect the desired performances, we have seen necessary to carry out some tests of robustness on which we have introduced some variations of motor parameters.

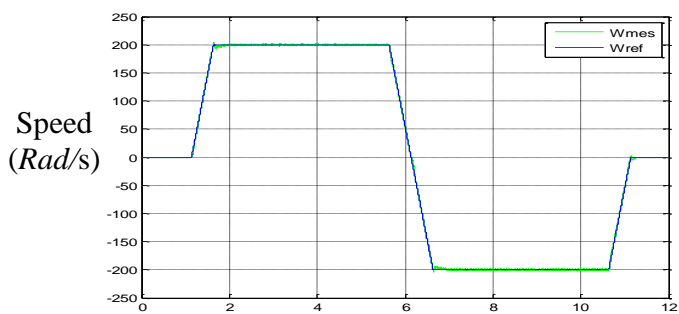

a)

time (s)

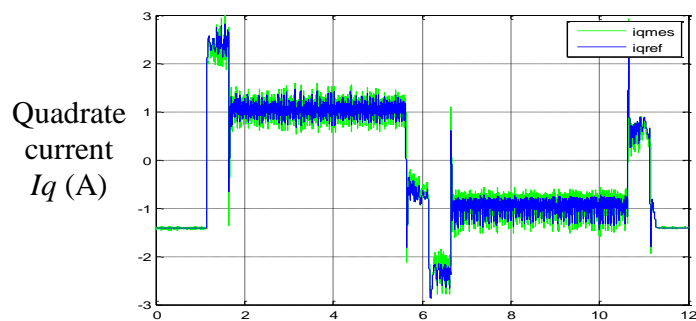

b)

Figure 17. Experimental results under modified IMC method: a) estimated speed, b) $I_{q}$ current with variation of inductances $L_{d q}$ of $20 \%$

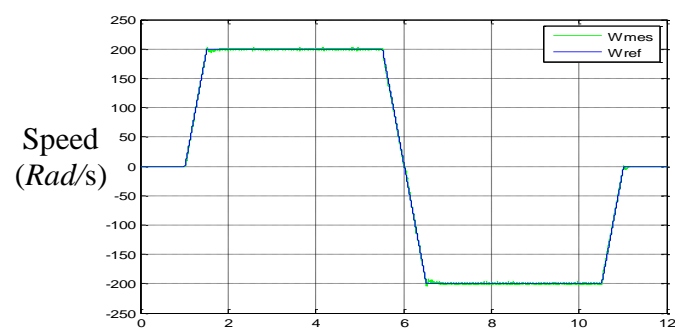

a) time $(s)$

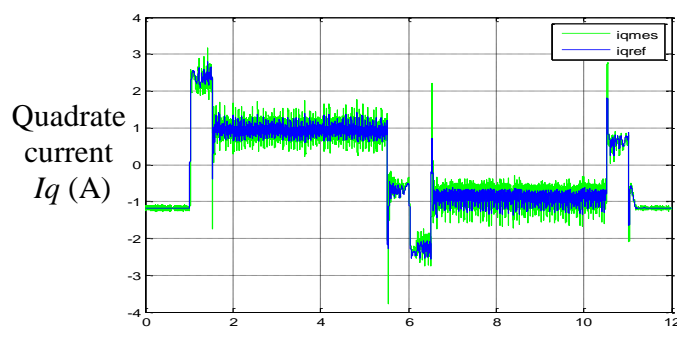

b) time $(\mathrm{s})$

Figure 18. Experimental results under modified IMC method: a) estimated speed, b) $I_{q}$ current with variation of inductances $L_{d q}$ of - $20 \%$ 


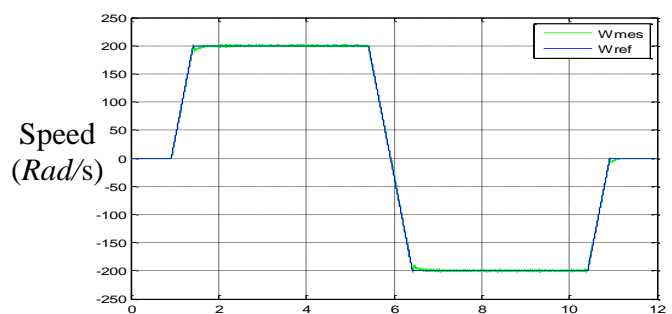

a)

time (s)

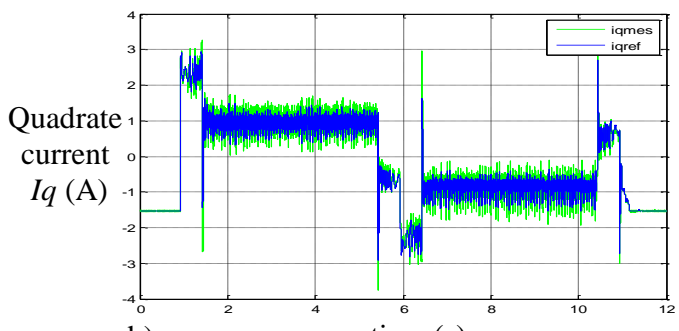

b) time (s)

Figure 19. Experimental results under modified IMC method: a) estimated speed,

b) $I_{q}$ current with variation of moment of inertia $J$ of $50 \%$

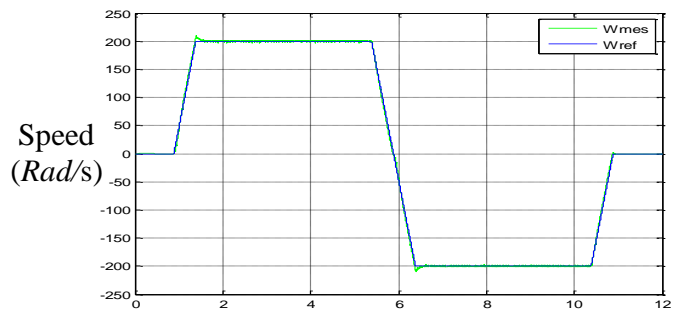

a) time (s)

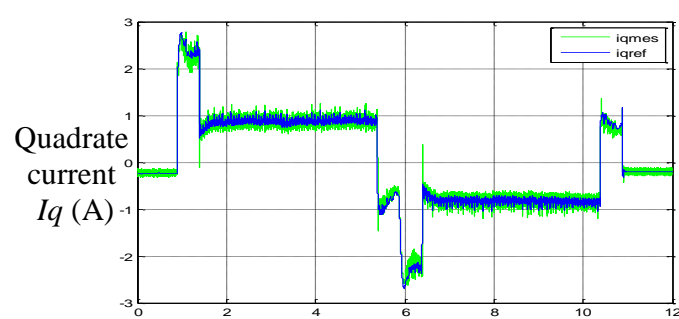

b) time (s)

Figure 20. Experimental results under modified IMC method: a) estimated speed,

b) $I_{q}$ current with variation of moment of inertia $J$ of $-50 \%$

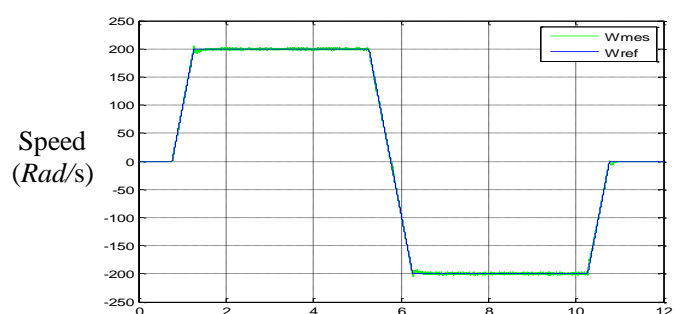

a)

time $(s)$

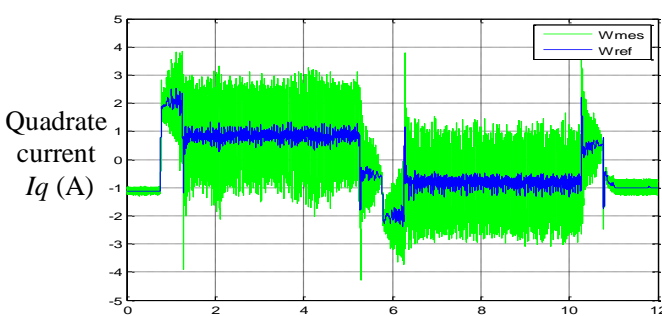

b)

time $(s)$

Figure 21. Experimental results under modified IMC method: a) estimated speed, b) $I_{q}$ current with variation of stator resistance Rs of $30 \%$

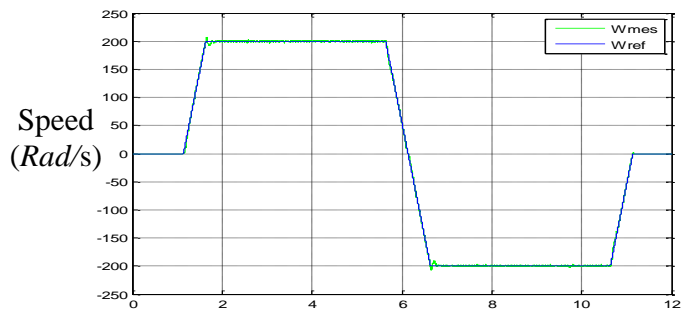

a) time (s)

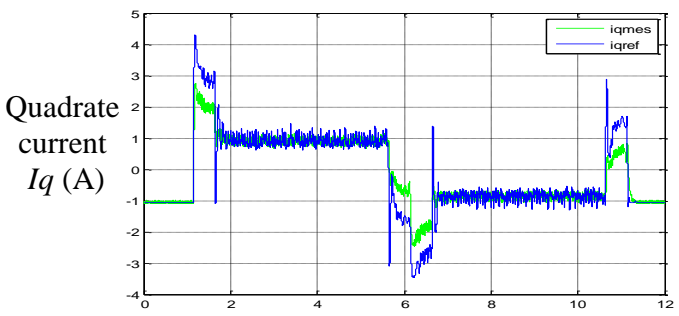

b) time $(s)$

Figure 22. Experimental results under modified IMC method: a) estimated speed, b) $I_{q}$ current with variation of stator resistance $R s$ of $-70 \%$ 


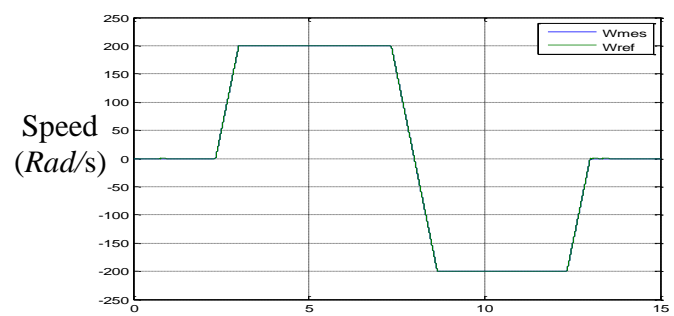

a)

time $(\mathrm{s})$

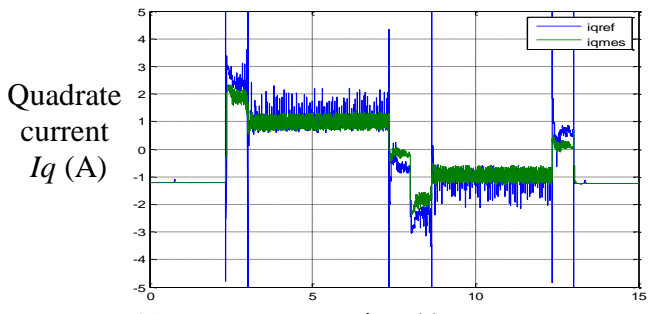

b)

time (s)

Figure 23. Experimental results under RST controller method: a) estimated speed, b) $I_{q}$ current with variation of inductances $L_{d q}$ of $50 \%$

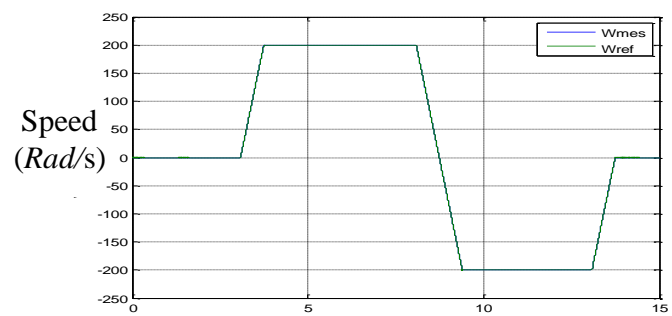

a)

time $(s)$

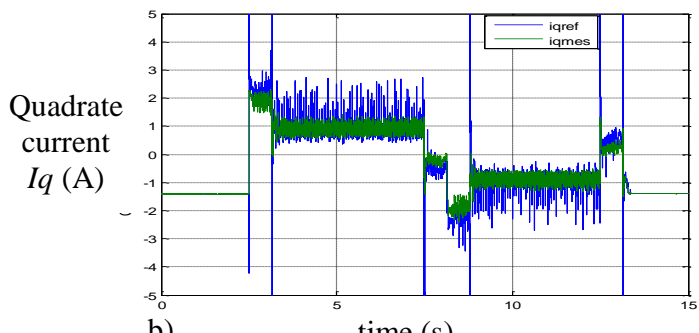

b)

Figure 24. Experimental results under RST controller method: a) estimated speed, b) $I_{q}$ current with variation of moment of inertia $J$ of $50 \%$

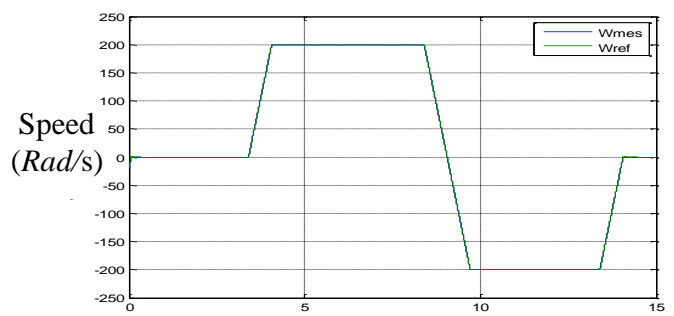

a) $\operatorname{timēes}(s)$

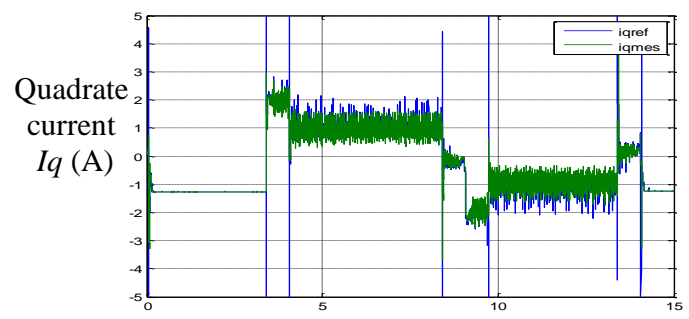

b)

time (s)

Figure 25. Experimental results under RST controller method: a) estimated speed, b) $I_{q}$ current with variation of stator resistance $R s$ of $100 \%$

Through these results, good robustness is observed in the response of the closed loop system with respect to parametric variations for the modified IMC method. The RST controller is more robust than the modified IMC one by the fact that there are no overshoot/undershoot in the shape of the speed response.

\section{Conclusion}

In this paper, we have developed two methods of control, namely the modified IMC and the RST controllers applied on a PMSM in order to regulate its speed and comparing the performance of each of them. Such controllers are adopted by the fact that they are largely used in industrial factories view to their simple implantation in microcontrollers. The study was done for the above PMSM in order to obtain high performances in terms of tracking and disturbances rejections. The two studied methods have present good results in both tracking and robustness towards parameters variations and load disturbance. Nevertheless, the IMC controller in spite of it's modified structure present some inflexibility. This is reflected in the presence of a little speed tracking error which is about of 0.2 and a little overshoot/undershoot. 
This disadvantage is almost absent in the response of the closed loop system provided by the RST controller.

\section{Acknowledgments}

The autors wish to thank all the team of the resarch Unit of Industrial Processes Control “ UIPC" in the National Engineering School of Sfax Tunisia which supported this work.

\section{References}

[1]. K. H. Kim and M.-J. Youn. "A nonlinear speed control for a PM synchronous motor using a simple disturbance estimation technique". IEEE Trans. on Industrial Electronics, 49: 524-535, 2002

[2]. T.H.Liu \& C.P.Cheng, "Controller design for a sensorless permanent magnet synchronous drives system". IEEE. Proceedings Electrical Power Applications Part B, vol.140 (6), pp 368-378

[3]. T. L. Hsien, Y. Y. Sun, and M. C. Tsai, "Hळ control for a sensorless permanent-magnet synchronous drive". IEE Proc.-Electr. Power Appl., 144: 173-181, 1997

[4]. P.M.Pelecezewski, W.Oberschelp, \& U.K.Kunz, "Optimal model-following control of a positioning drive system with a permanent-magnet synchronous motor". IEEE Proceeding Control Theory and Applications, Part-D, vol 138 (3), pp 267-273, 1991

[5]. Y. A.-R. I. Mohamed, "Design and implementation of a robust current control scheme for a PMSM vector drive with a simple adaptive disturbance observer". IEEE Trans. on Industrial Electronics, 54: 1981-1988, 2007

[6]. N.Matsui., \& H.Ohashi, "DSP-based adaptative control of a brushless motor". IEEE Transactions on Industry Applications, vol 28 (2), pp 448-454, 1992.

[7]. E.Cerruto, A.Consoli, A.Racitti, \& A.Testa, "A robust adaptative controller of PM motor drives in robotic applications". IEEE Transactions on Power Electronics, vol 10 (1), pp 62-71, 1992

[8]. N.Hemati, J.S.Thorp \& M.C.Leu, "Robust nonlinear control of brushless DC motors for direct-drive robotic applications", IEEE Transactions on Industrial Electronics, vol 37 (6), pp 460-468, 1990

[9]. I. C. Baik, K.-H. Kim, and M. J. Youn, "Robust nonlinear speed control of PM synchronous motor using boundary layer integral sliding mode control technique", IEEE Trans. on Control Systems Technology, 8: 47-54, 2000

[10]. F. J. Lin, C. H. Lin, "A permanent magnet synchronous motor servo drives using selfconstructing fuzzy neural network controller", IEEE Trans. on Energy Conversion, 19: 66-72, 2003.

[11]. C. E. Garcia, M. Morari, "Intenal model control-1:a unifying review and some new results", Industrial Engineering Chemistry Process Design and Development, 21: 308323, 1982

[12]. M. Morari, E. Zafirious, “Robust Process Control”, New Jeresy: Prentice-Hall, 1989.

[13]. L. Harnefors, H. P. Nee, "Model-based current control of AC machines using the internal model control method", IEEE Trans on Industry Applications, 34: 133-141, 1998.

[14]. I. G. Horn, J. R. Arulandu, R. D. Braatz, "Improved filter design in internal model control", Industrial Engineering Chemistry Research, 35: 3437-3441, 1996

[15]. A. Zheng, M. V. Kothare, M. Morari, "Anti-windup design for internal model control", International J of Control, 60: 1015-1024, 1994

[16]. H. Gharsallaoui, M.Ayadi, M.Benrejeb, P.Borne, "Flatness-based control and conventional RST polynomial controlof a thermal process", Int. J of Computers, Communications \& Controls, ISSN 1841-9836, E-ISSN 1841-9844 Vol. IV (2009), No. 1, pp. 41-56

[17]. Bendjedia, K. Tehrani, and Y. Azzouz, "Design of RST and fractional order PID controllers for an induction motor drive for electric vehicle application," 2014 
[18]. M. Khanchoul and M. Hilairet, “ Design and comparison of different rst controllers for pmsm control," IECON 2011-37th Annual Conference on IEEE Industrial Electronics Society. IEEE,2011, pp.1795-1800

[19]. J. Khedri, M.Chaabane, M.Souissi, "Speed control of a permanent magnet synchronous machine powred by voltage inverter: Moment approach", Int. J of Computers, Communications \& Controls, ISSN 1841-9836, E-ISSN 1841-9844 Vol. VI (2011), No. 1 (March), pp. 90-100

[20]. J. Khedri, M.Chaabane, M.Souissi, D.Mehdi, "Speed control of a permanent magnet synchronous machine (PMSM) fed by volatge inverter fuzzy control approach", Int. J of Electrical and Information Engineering 4.42010

[21]. J. Khedri, M.Chaabane, M.Souissi, "Robust control of a permanent magnet synchronous machine (PMSM) using internal model control", Proceedings of the International Technology Management Conference Antalya, Turkey 2015

[22]. Shihua Li, Senior Member, IEEE, Hao Gu,"Fuzzy Adaptive Internal Model Control Schemes for PMSM Speed-Regulation System", IEEE Transactions On Industrial Informatics, Vol. 8, No. 4, November 2012

[23]. C. Eric, "Internal model predictive control," Automatica, vol. 31, no.10, pp. 1471-1482, 1995.

[24]. F. J. Lin and C. H. Lin, “A permanent magnet synchronous motor servo drives using selfconstructing fuzzy neural network controller," IEEE Trans. Energy Convers, vol. 19, no. 1, pp. 66-72, Jan. 2003.

[25]. D. Landau, G. Zito, "Digital Control Systems: Design, Identification and Implementation". Springer,2006

[26]. Ostertag, E, E. Godoy, "RST-controller design for sinewave references by means of an auxiliary Diophantine equation", Proc. 44th IEEE Conference on Decision and Control and European Control Conference, CDC-ECC'05, pp. 6905-6910, Seville

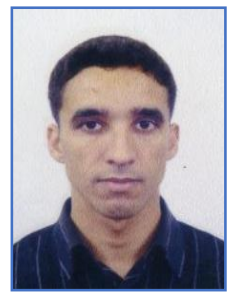

Jamel Khedri received the Ph. D degree from National School of Engineers of Sfax (ENIS), University of Sfax, Tunisia, in 2010. His currents research interests robust control, fuzzy logic control, D-stability analysis and applications of these techniques to induction motors

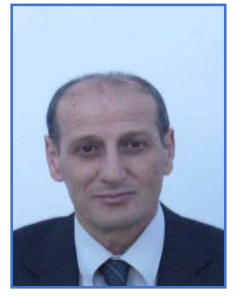

Mansour SOUISSI received his $\mathrm{Ph}$. D in Physical Sciences from the University of Tunis in 2002. He is Professor in Automatic Control at Preparatory Institute of Engineers of Sfax, Tunisia. Since 2003, he is holding a research position at Automatic Control Unit, National School of Engineers of Sfax, Tunisia. His current research interests robust control, optimal control, fuzzy logic, linear matrix inequalities and applications of these techniques to agriculture systems. Dr Souissi is a member of the organization committees of several national and international conferences (STA, CASA) 